\title{
АНАЛИЗ ИННОВАЦИОННОГО РАЗВИТИЯ ПРОВИНЦИЙ КНР НА ОСНОВЕ ЧЕТЫРЕХКОМПОНЕНТНОЙ РЕГРЕССИОННОЙ МОДЕЛИ
}

\author{
() 2021 Вэнь Юйчжу \\ директор строительной компании ООО «Синчжу», \\ внештатный сотрудник международного отдела Shenyang Jianzhu University, KHP \\ E-mail: wenyuzhu1983@163.com
}

(c) 2021 Санжина Ольга Петровна

доктор экономических наук, профессор кафедры «Управление инвестициями и недвижимостью» Восточно-Сибирский государственный университет технологий и управления,

Респ. Бурятия, Улан-Удэ

E-mail: osanzhina@yandex.ru

(c) 2021 Аюрзанайн Аюр Биликтоевич

кандидат экономических наук, доцент кафедры «Экономическая теория, мировая экономика, государственное и муниципальное управление»

Восточно-Сибирский государственный университет технологий и управления,

Респ. Бурятия, Улан-Удэ

E-mail: ayurza9@yandex.ru

(ㄷ) 2021 Дармаева Оксана Александровна

магистрант кафедры «Экономика, организация и управление производством»

Восточно-Сибирский государственный университет технологий и управления,

Респ. Бурятия, Улан-Удэ

E-mail: oksana.smart@yandex.ru

Данная статья посвящена анализу развития инновационных систем в регионах КНР (среди которых выбрано 30 китайских провинций). Авторы отмечают, что региональные результаты инновационной деятельности представляют собой совокупность моделей поведения отдельных лиц или организаций в отношении процесса производства инноваций. Однако, в данном исследовании основное внимание уделяется влиянию региональных институциональных и экономических рамок или условий на региональный инновационный потенциал. Китай на сегодня является одним из лидеров с точки зрения темпов развития национальной инновационной системы, что делает анализ развития региональных инновационных систем актуальной задачей экономической науки на современном этапе.

Ключевые слова: Китай, инновации, национальная инновационная система, региональная инновационная система, регрессионный анализ, корреляция Пирсона, коэффициенты регрессии, моделирование.

Для анализа развития инновационных систем в регионах КНР выбрано 30 китайских провинций в качестве единиц анализа. Богатый панельный набор данных за пять лет, в основном за первые 5 лет вступления в 21 век, используется для поддержки нашего эмпирического исследования и исследования периодического эффекта развития региональных инновационных систем Китая [1] за 10-й пятилетний период. Источники всех показателей измерения приведены в таблице 1. Статистические наборы данных показателей измерения экзогенных конструк-

ций относятся к периоду с 2001 по 2005 год, за исключением CU_K_STO, в то время как наборы данных INNO_OUT относятся к периоду с 2003 по 2007 год с временным лагом в два года. Поскольку ежегодные статистические данные собираются в конце каждого года, мы используем статистические наборы данных за период 20002004 годов для четырех показателей измерения CU_K_STO. В совокупности мы получили 150 единиц наблюдений в панельном наборе данных за год провинции, который не только предоставляет достаточное количество выборок для эффек- 
тивной оценки параметра, но также использует преимущества как поперечного сечения, так и вариаций временных рядов. Единичный анализ, основанный на наборах данных региона, также может выявить основные причины, приводящие к различиям в региональном инновационном потенциале. Несомненно, региональные результаты инновационной деятельности представляют собой совокупность моделей поведения отдельных лиц или организаций в отношении процесса производства инноваций. Однако в данном исследовании основное внимание уделяется влиянию региональных институциональных и экономических рамок или условий на региональный инновационный потенциал. Таким образом, измерение совокупности различных элементов на уровне отдельных лиц или организаций даст частичный ответ на вопрос о том, как и почему существуют региональные различия в инновационном потенциале.

Для эффективной оценки модели некоторые наборы данных нуждаются в повторной обработке. Сначала мы дефлируем все данные о расходах до значения 2000 года в анализе. Кроме того, мы преобразуем эти непосредственно наблюдаемые значения показателей измерения в логарифмические значения из-за асимметрии их распределения, что лучше согласуется с предположениями о распределении процедуры оценки. Это менее чувствительно к выбросам и согласуется с предыдущими исследованиями в этой области (например, Гриличес, 1990; Фурман, Портер и Стерн, 2002) [2].

В таблице 1 представлена описательная статистика наборов данных измерений и их коэффициентов корреляции Пирсона. Почти все коэффициенты значимы на уровне 1\% теста, и многие значения коэффициентов превышают 0,9. Таким образом, существует серьезная коллинеарность между 4 объясненными переменными и 22 исследовательскими переменными. Поэтому рекомендуется использовать моделирование PLS для работы с нашими скомпилированными наборами данных. В частности, мы применяем SIMCA-P v10.0 для регрессии PLS и SmartPLS v2.0 для моделирования пути PLS в нашем эмпирическом исследовании.

Что касается взаимосвязи между первым компонентом (объясненная дисперсия 91\%) зависимых переменных и первым компонентом (объясненная дисперсия 85\%) независимых переменных с помощью SIMCA-P, очевидно, це- лесообразно использовать линейное уравнение без выбросов для описания взаимосвязи между эндогенными инновационными выходными переменными и объясняющими инновационными детерминантами в анализе. Четырехкомпонентная регрессионная модель PLS автоматически получается с R2X=0,892, R2Y=0,933 и Q2 (перекрестно проверенный R2) = 0,922, что указывает на лучшую способность объяснения и качество прогнозирования выбранной нами модели. Значение переменной в прогнозе (VIP), которое суммирует важность исследуемых переменных, является совокупной мерой влияния переменной. Результаты VIP исследовательских переменных, основанные на наших эмпирических наборах данных, показаны на Рисунке. Исследовательское исследование показывает, что VIP-персоны почти всех исследовательских переменных превышают 0,7. Это означает лучшее соответствие выбранных исследовательских переменных в нашей системе показателей.

В частности, три фондовых индикатора, CU_D_PAT, CU_D_PAP и CU_I_PAP, отображают доминирующий вклад в INNO_OUT. Другими важными показателями (VIPN1.0), следующими за ними, являются A\&E_RD, S\&E_FTE, UNI_RD_P, GOV_S\&T, TMI_RD_P, B_RD, PRI_RD_P, HTI_RD_P и DE_CA, в свою очередь. Доминирующая роль фондовых показателей с выделенным вкладом S\&E_FTE в качестве инвестиций в человеческий капитал указывает на то, что производственная модель региональных инноваций Китая в целом характеризуется функцией производства оригинальной идеи Ромера, где прирост новых знаний положительно зависит от совокупного запаса знаний и объема человеческого капитала, занятого в НИОКР. Кроме того, более очевидная важность A\&E_RD, чем B_RD, в показателях региональной инновационной продукции подтверждает доминирующую роль прикладных и экспериментальных исследований и разработок и относительно более слабую роль базовых исследований и разработок в региональных инновациях. Относительно выдающиеся результаты UNI_RD_P показывают, что университеты играют важную роль в улучшении регионального инновационного производства. Значительная важность GOV_S\&T означает, что ряд государственных программ, таких как программа скалолазания, программа 863 и программа Torch, оказывают явное стимулирующее влияние на региональный инновационный выпуск в Китае. 


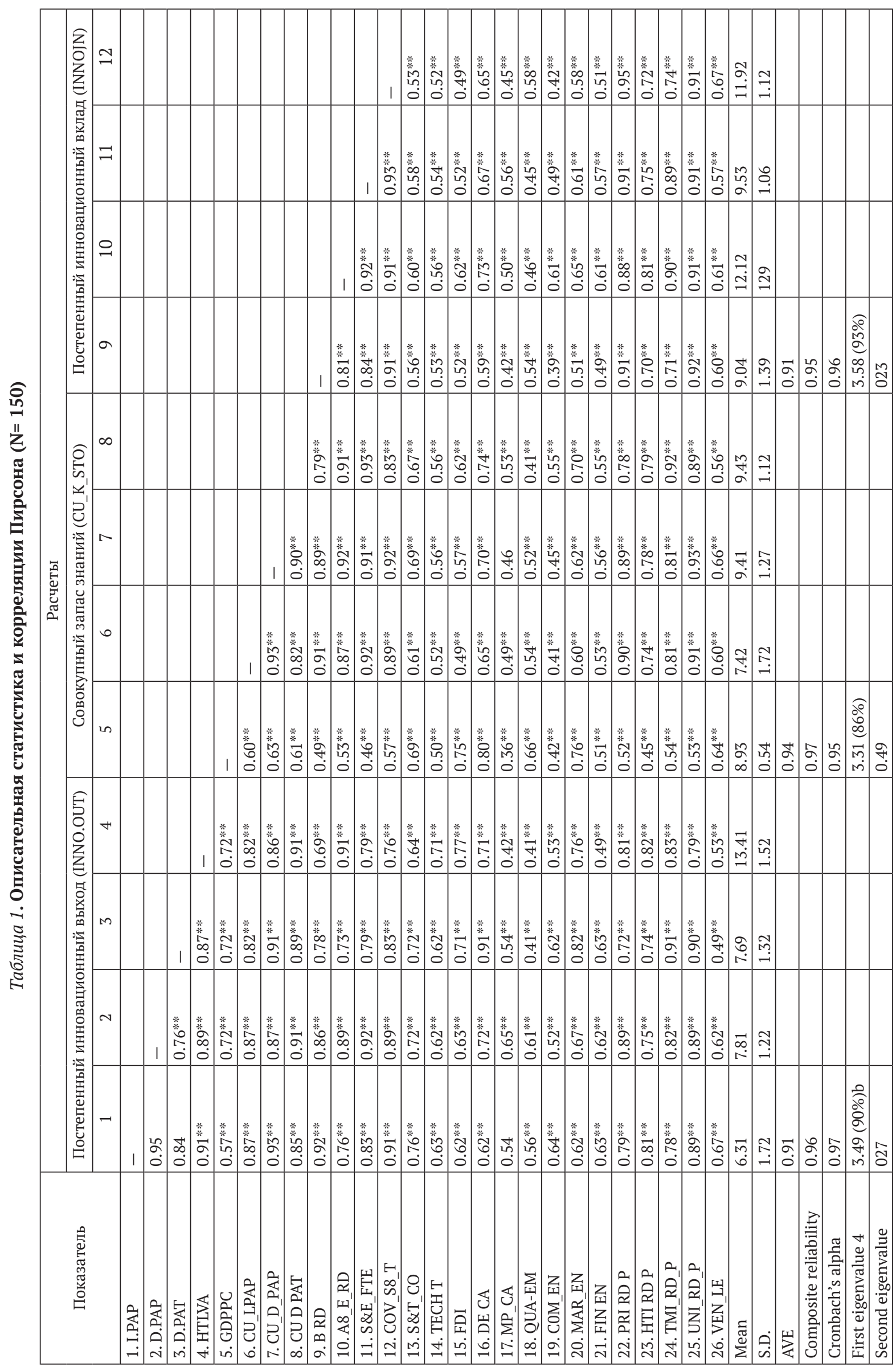




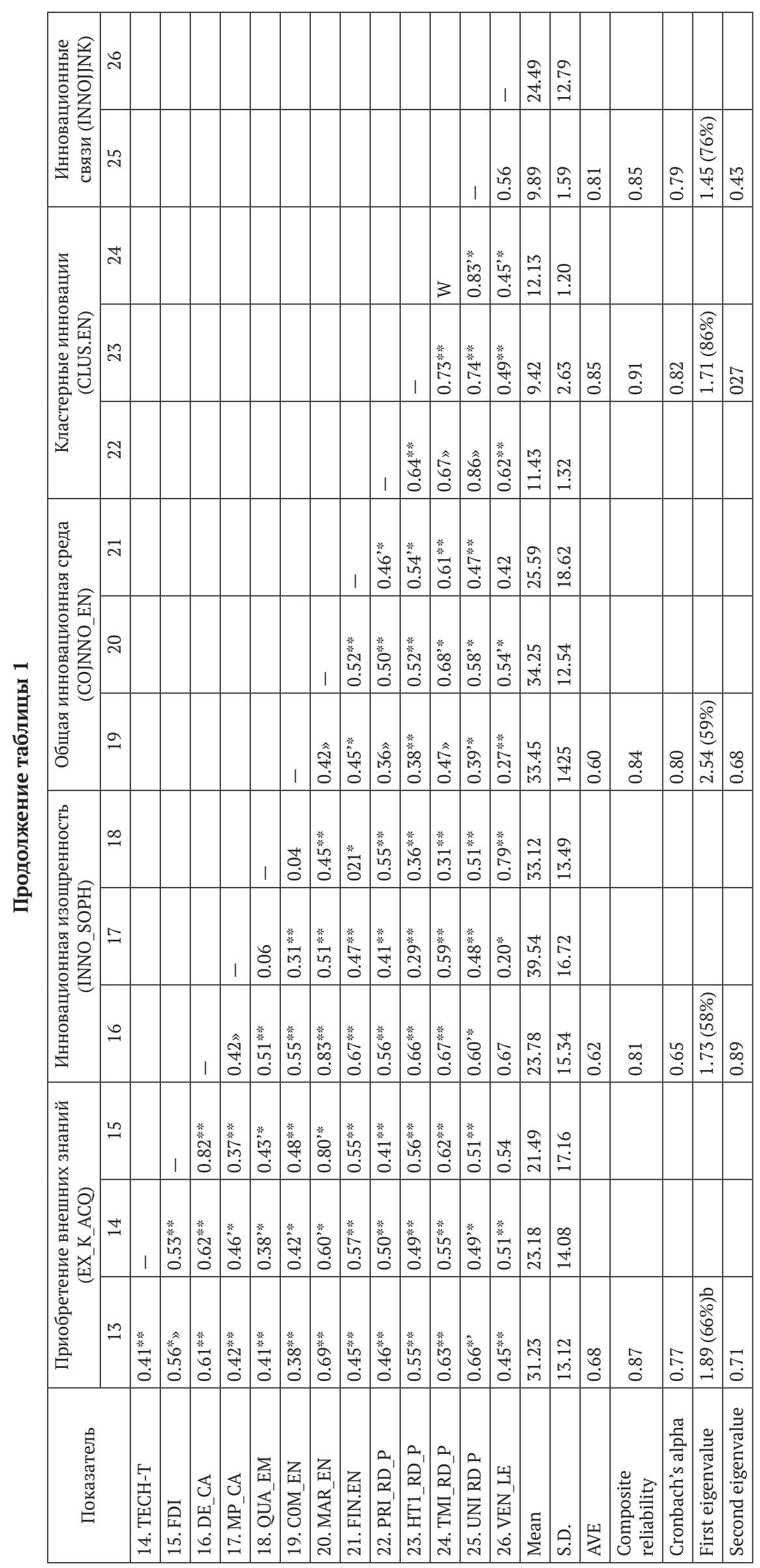


Кроме того, относительно удовлетворительные значения VIP показателей CLUS_EN (TMI_RD_P и HTI_RD_P) показывают, что специфичная для кластера среда демонстрирует активное продвижение китайского регионального инновационного производства. В исключительных случаях GDPPC относительно показывает меньший вклад в INNO_OUT, что кажется парадоксом со значительными корреляциями между ним и показателями измерения INNO_OUT (таблица 2). Разумная интерпретация состоит в том, что влияние GDPPC как показателя уровня экономики региона или запаса знаний на INNO_OUT «вытесняется» относительно более доминирующими эффектами таких важных показателей, как CU_I_PAP, CU_D_PAP, CU_D_PAT, S\&E_FTE и A\&E_RD.

C точки зрения вклада каждого исследовательского показателя в отдельные четыре показателя инновационной продукции (D_PAT, HTI_VA, I_PAP и D_PAP), мы обнаружили, что существуют значительные различия.

В порядке убывания важности CU_D_PAT, HTI_RD_P, TMI_RD_P и ПИИ отображают доминирующий вклад в выпуск D_PAT, в то время как HTI_RD_P, CU_D_PAT, ПИИ и TMI_RD_P доминируют в размере HTI_VA. Очевидный вклад ПИИ в технологические инновации подтверждает вывод, сделанный Ченгом и Лином (Cheung and Lin, 2004) [3]. В отношении научной инновационной продукции, т.e.I_PAP, D_PAP, B_RD, PRI_RD_P, UNI_ RD_P и GOV_S\&T, показывают активный вклад, за исключением вклада совокупного запаса знаний, встроенного в CU_I_PAP и CU_D_PAP.

Для рефлексивных конструкций сначала следует оценить конвергентную и дискриминант- ную валидность. Путем оценки в соответствии с программой SmartPLS мы получили высокую загрузку и вес каждой переменной манифеста на соответствующей конструкции, в соответствии с которой приводится t-статистика с помощью процедуры повторной выборки начальной загрузки. Значения загрузки всех переменных манифеста превышают 0,80 (большинство превышает 0,90), поэтому дискриминантная валидность каждой переменной манифеста лучше. Т-статистика загрузки каждой переменной манифеста в соответствующую конструкцию значительна, что показывает лучшую сходимость всех переменных манифеста.

Затем проверяется одномерность каждой конструкции. Статистика альфа Кронбаха в таблице 2 приводит к признанию одномерности всех конструкций, за исключением конструкции INNO_SOPH (альфа Кронбаха должна превышать рекомендуемый минимум 0,70). Это подтверждает надежность взаимосвязей между большинством измеряемых переменных и скрытыми переменными. Кроме того, с точки зрения анализа главных компонент (РСА), первое собственное значение корреляционной матрицы всех переменных манифеста для каждой конструкции больше 1, а второе меньше 1 . В то же время результаты РСА показывают, что на первый фактор приходится 58-93\% дисперсии в соответствующих наборах данных конструктов, что позволяет предположить, что один фактор может полностью объяснить большую часть дисперсии.

Одной только одномерности недостаточно для обеспечения полезности шкалы, и надежность сводной оценки следует оценивать по-

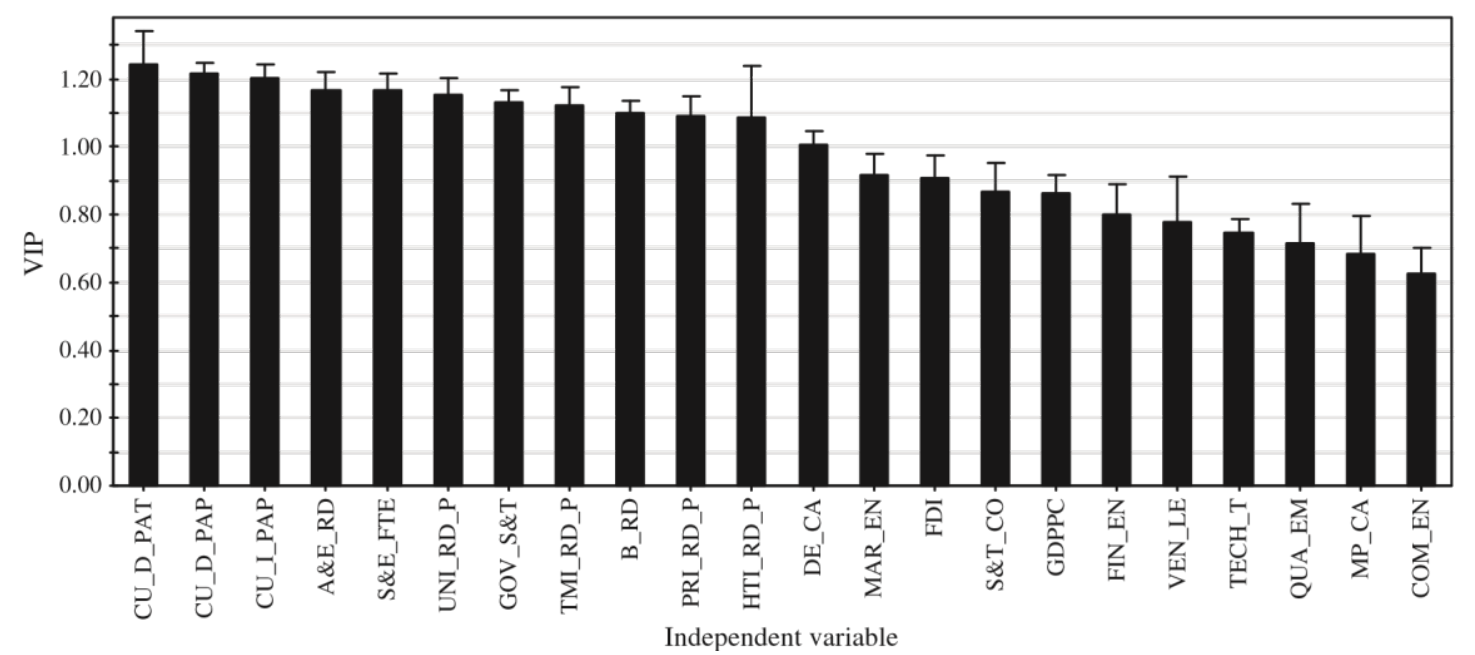

Puc. 1. Распределение независимых переменных в четырехкомпонентной регрессионной модели PLS 


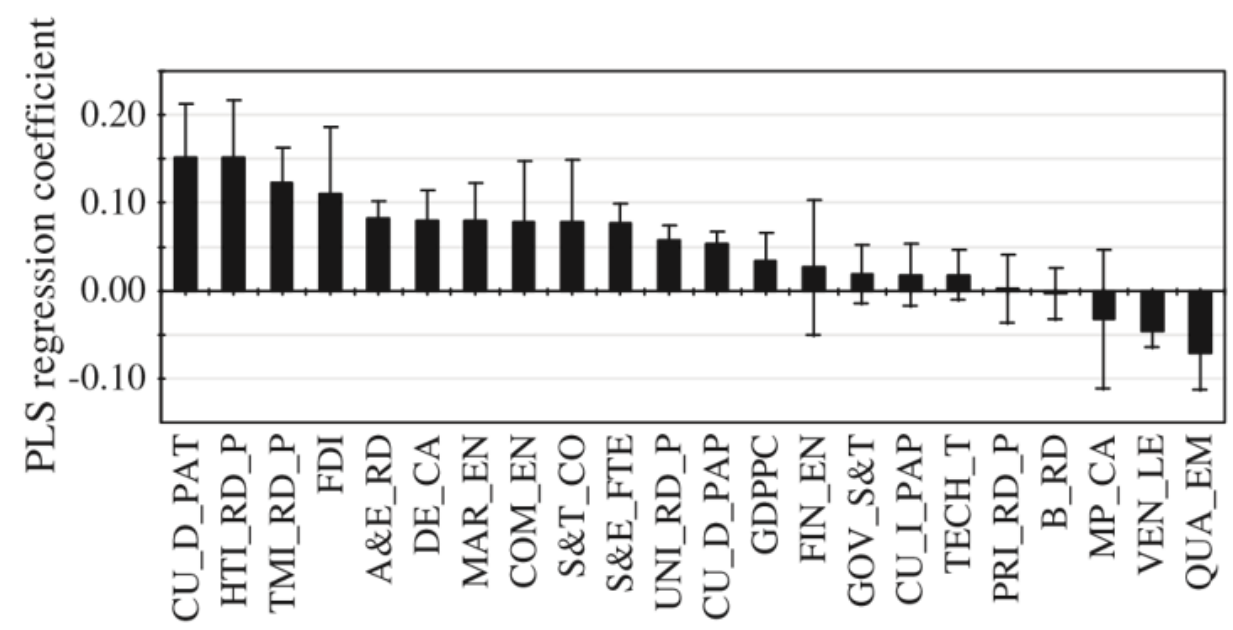

Puc. 2. (D_PAT в качестве зависимой переменной) Скорректированный RX2=0,915 Q2 =0,902

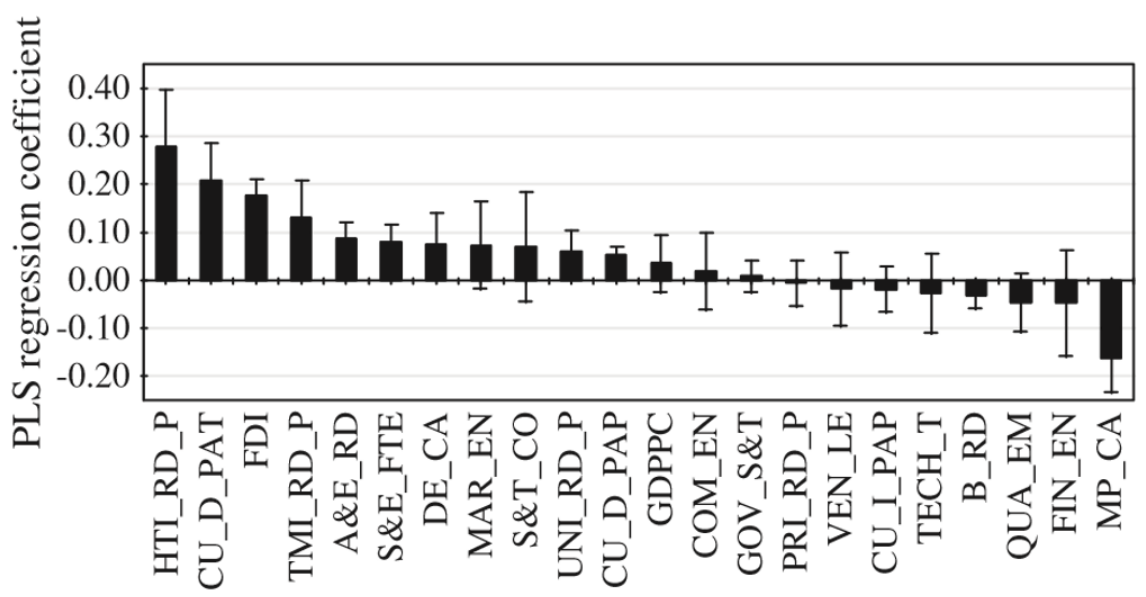

Puc. 3. (HTI_VA в качестве зависимой переменной) Скорректированный RX2=0,913 Q2=0,907

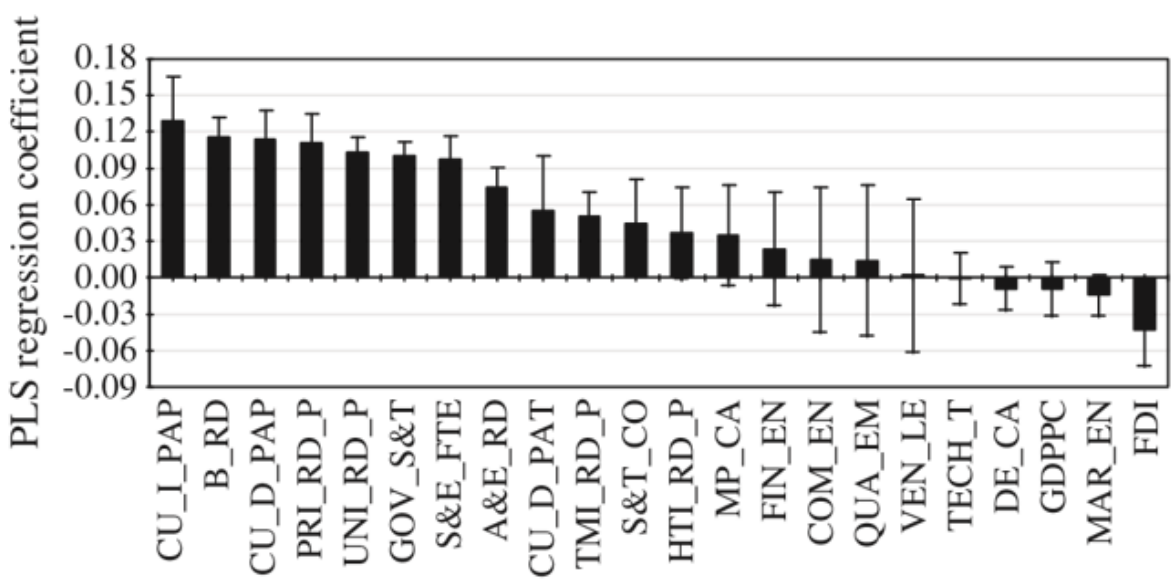

Independent variable

Puc. 4. (I_PAP в качестве зависимой переменной) Скорректированный RX2=0,920 Q2=0,919 


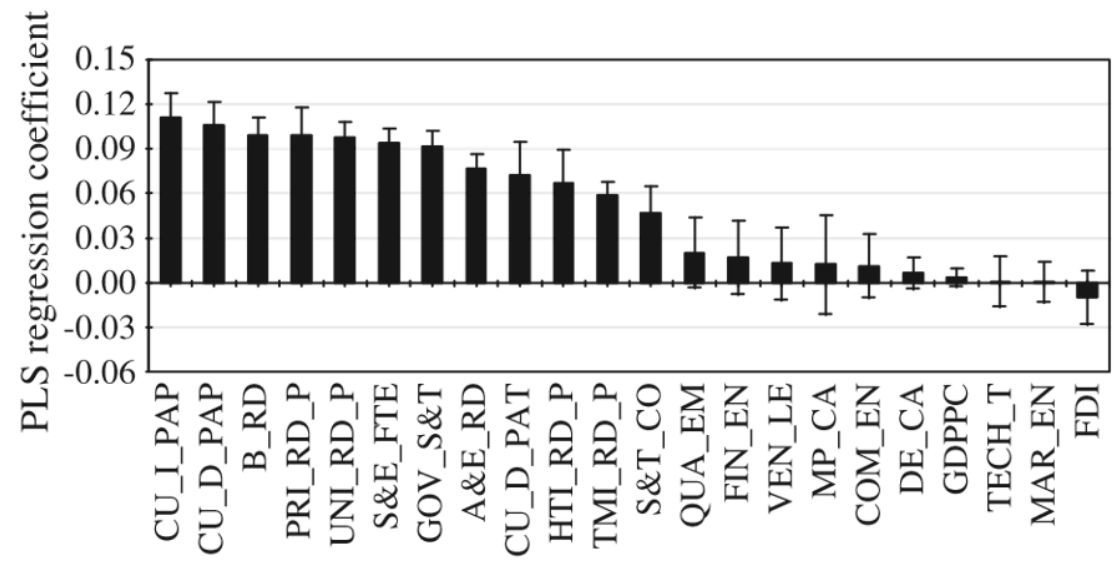

Independent variable

Puc. 5. (D_PAP в качестве зависимой переменной) Скорректированный RX2=0,953 Q2=0,952

сле того, как одномерность будет приемлемо установлена. Для оценки надежности скрытой переменной, т.е. оценки того, насколько точны оценки пути для «истинного» эффекта. Это лучший показатель оценки надежности, чем альфа Кронбаха, который, предполагая параллельные измерения, представляет собой оценку внутренней согласованности с нижней границей. Значение надежности композитов для наших конструкций удовлетворяет минимальному значению 0,70 .

Кроме того, SmartPLS предоставляет результат извлечения средней дисперсии (AVE) для каждой конструкции, который также называется «индексом общности» и используется для измерения качества модели измерения для каждой конструкции. AVE является дополнительной мерой для значения надежности конструкции, и принимается значение выше 0,50 для AVE любой конструкции. В этом эмпирическом анализе все оценки превышают критическое значение $(0,50)$, что является дополнительным доказательством надежности.

Сначала мы исследуем основной причинноследственный эффект между INNO_OUT и CU_K_ STO или INNO_IN на основе производственной функции идеи Ромера (1990) [4] (IPF). Затем в эту базовую структуру пути добавляются или выбираются другие экзогенные конструкции для углубленных исследований. Таким образом, можно получить более ценную информацию, такую как взаимозависимость и взаимодополняемость между инновационными детерминантами.

В таблице 2 представлен широкий спектр результатов, касающихся случайных взаимосвязей между инновационной выходной конструкцией (INNO_OUT) и различными инновационными входными конструкциями (CU_K_STO, INNO_IN и EX_K_ACQ), а также ее движущими или вспомогательными конструкциями (INNO_SOPH, CO_INNO_EN, CLUS_EN и INNO_LINK). Эти подтверждающие результаты дают стабильные доказательства того, что большинство функциональных конструкций в RISS Китая работают хорошо, за исключением NNO_LINK и INNO_SOPH. Это показывает, что базовая функциональная структура РИС Китая сформировалась, но не является совершенной.

Во-первых, мы реализуем базовый анализ путей для исследования основных функций в RIS, созданных тремя инновационными входными конструкциями, CU_K_STO, INNO_IN и EX_K_ACQ, в выходной конструкции INNO_OUT. В первой модели пути 4.1 мы обнаруживаем, что конструкция CU K STO может интерпретировать 95,5\%-ную дисперсию конструкции INNO_ OUT и оказывает на нее очень значительное положительное влияние. Значительное влияние инкрементного INNO_IN на INNO_OUT также обнаружено в модели пути 4.2. Однако ее прогноз менее точен, и интерпретация 5,2\% - ной дисперсии теряется по сравнению с моделью пути 4.1. Модель пути 4.3 показывает, что CU_K_STO и INNO_IN вместе предсказывают лучший прогноз (R2=0,966). Это еще раз доказывает, что модель эндогенного роста Ромера возникает и развивается на региональном инновационном производстве в Китае. Тем не менее, все еще существует 3,4\% - ная дисперсия INNO_OUT, которую необходимо интерпретировать путем дополнительного рассмотрения таких экзогенных 


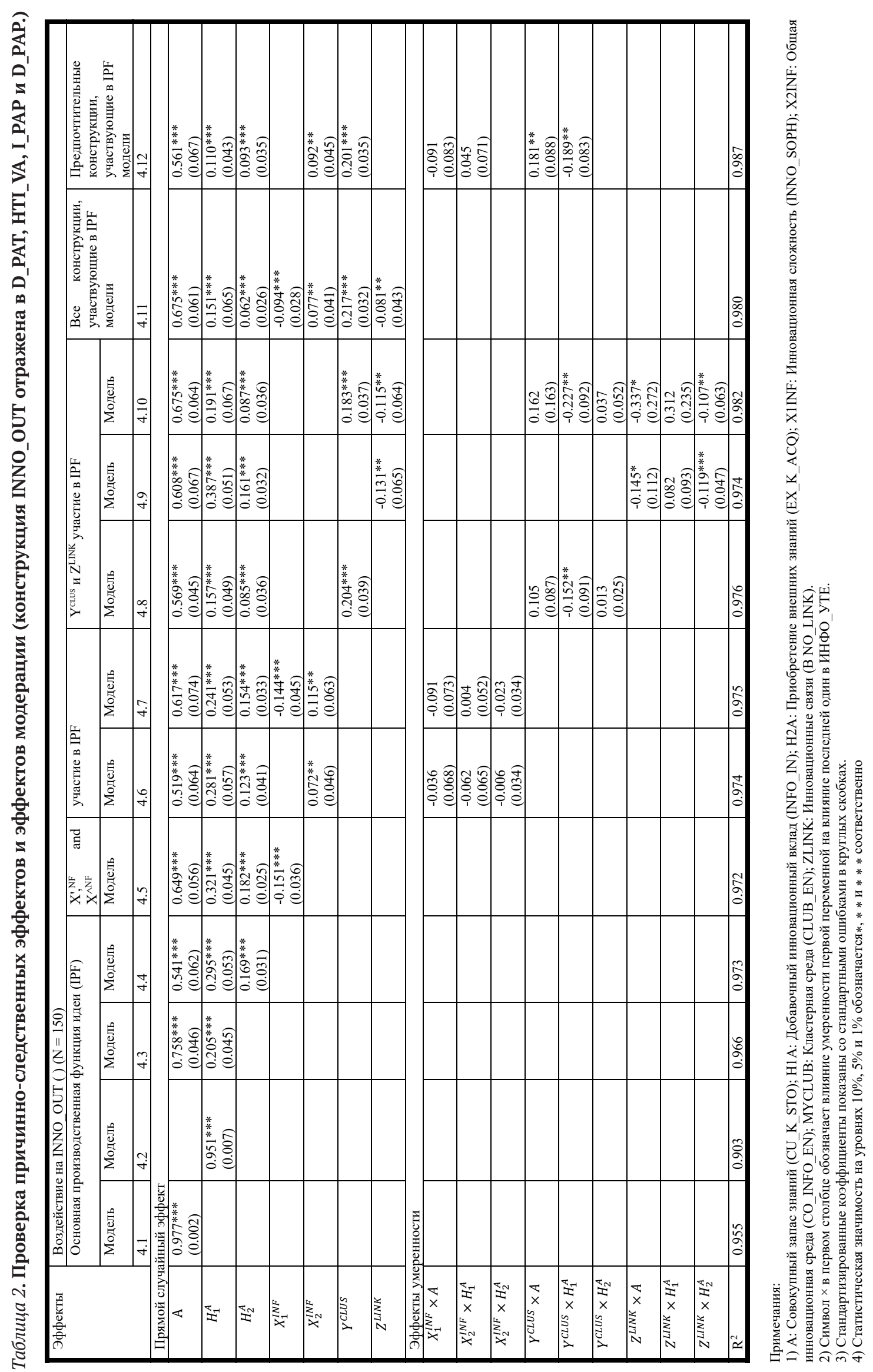


движущих или поддерживающих элементов. В модели пути 4.4 мы включаем EX_K_ACQ в нашу структуру на основе модели 4.3. Подтверждающие результаты, представленные в таблице 2, показывают, что было интерпретировано большее отклонение INNO_OUT (R2=0,973), и в то же время EX_K_ACQ показывает статистически значимое и положительное влияние на INNO_OUT на уровне 1\%. Это означает, что регионы с более высоким уровнем научно-технического сотрудничества и передачи знаний между участниками и более высоким уровнем ПИИ должны иметь возможность получать значительно более высокий объем научно-технической инновационной продукции. Сравнивая стандартизированные коэффициенты пути, полученные с помощью модели пути 4.4, становится ясно, что региональный CU_K_STO показывает наиболее доминирующий вклад в региональный инновационный выпуск. INNO_IN следует за ним, а EX_K_ACQ находится на последнем месте. Однако, в отличие от результатов, полученных в модели пути 4.3 без EX_K_ACQ, доминирующий статус CU_K_STO был ослаблен, но влияние INNO_ IN на инновационную продукцию было усилено в модели пути 4.4. Это означает, что EX_K_ACQ выгоден для улучшения новых дополнительных инновационных ресурсов путем стимулирования и, следовательно, помогает регионам в некоторой степени избавиться от независимости от предыдущего CU_K_STO.

Затем мы обратимся к трем указанным моделям путей (4.5-4.7) для углубленного изучения случайных эффектов INNO_SOPH и CO_INNO_EN на INNO_OUT на основе модели 4.4. INNO_SOPH показывает статистически значимое и отрицательное влияние на INNO OUT, в то время как CO INNO EN показывает слабо положительное существенное влияние на INNO_OUT при исследовании соответственно на уровне 5\%. Причина, объясняющая неожиданное влияние INNO_SOPH на региональное инновационное производство, многообразна. Этот вывод подтверждает слабый инновационный потенциал китайских предприятий. Фактом, которым нельзя пренебрегать, является нехватка высококвалифицированных и ориентированных на инновации технических специалистов, и технических работников в Китае (ОЭСР, 2008). Одна из основных причин заключается в том, что китайская система образования ориентирована на пассивное обучение и успеваемость на основе экзаменов (ОЭСР, 2008), что заставляет студентов терять инновационное мышление и креативность. Кроме того, межрегиональная разница в специальном распределении интеллектуального капитала никогда не исчезает со статистически неожиданным результатом. С точки зрения возможности интерпретации отклонений INNO_OUT, INNO_SOPH не улучшает моделирование пути (R2=0,972), в то время как CO_INNO_EN работает немного лучше (R2=0,974) по сравнению с моделью 4.4. Когда две экзогенные конструкции вместе включены в модель 4.4, т.е. формируется новая модель пути 4.7, качество интерпретации коэффициентов пути почти не меняется (R2=0,975). INNO_SOPH по-прежнему демонстрирует статистически значимое и негативное влияние на INNO_OUT, в то время как CO_INNO_EN демонстрирует статистически значимое и положительное влияние на INNO_OUT на уровне $5 \%$.

Далее мы приступаем к оценке относительной роли как INNO_LINK, так и CLUS_EN в региональном инновационном производстве. Основываясь на результатах исследования группы моделей путей 4.8-4.10, ясно, что обе они оказывают значительное влияние на INNO_OUT, в то время как эффект, создаваемый INNO_LINK, отрицателен. Это означает, что связующие эффекты UNI_RD_P и VEN_LE между общей инновационной инфраструктурой и CLUS_EN плохо работают с точки зрения РИС Китая. Вспомним Рисунок 2 и 5, очень низкая значимость VEN_LE в INNO_OUT может интерпретировать этот неожиданный результат. Более того, неудивительно получить этот неудовлетворительный результат из-за различных практических ограничений. Например, финансовая система Китая не удовлетворяет потребности в финансировании частных фирм, особенно малых и средних предприятий, и ощущается острая нехватка капитала для финансирования новых предприятий, которые являются одним из важных источников инноваций [5] (ОЭСР, 2008). В свете статистически значимого и положительного влияния CLUS_EN на INNO_OUT на среднем региональном уровне этот вывод подразумевает, что относительно более высокие уровни концентрации спецификации отраслевого кластера и масштаба отраслевых НИОКР могут быть связаны с более высоким уровнем инновационной продукции благодаря инновационному сотрудничеству, передаче знаний или конкурентоспособности. С точки зрения качества прогнозирования модели 
путей 4.8-4.10 показывают лучшие результаты, чем другая группа моделей путей 4.5-4.7. Как видно из значений R2 в таблице 2, модели путей 4.8-4.10 интерпретируют большую дисперсию INNO_OUT.

Модель пути 4.11 включает все конструкции в интегрированную структурную структуру. В соответствии с результатами соответствующих экзогенных конструкций, все конструкции оказывают значительное влияние на INNO_OUT на уровне $1 \%$ или 5\%, в то время как INNO_LINK и INNO_SOPH по-прежнему демонстрируют негативные эффекты. Надежное сохранение положительных и статистически значимых эффектов CU_K_STO, INNO_IN, EX_K_ACQ, CO_INNO_EN и CLUS_EN на INNO_OUT во всех моделях альтернативных путей отражает ключевые детерминанты продвижения региональной инновационной продукции Китая. Модель 4.12 включает эти ключевые детерминанты в нашу модель предпочтительного пути, и было получено наилучшее качество прогнозирования модели (R2=0,987). Каждый из включенных коэффициентов траектории оказывает значительное влияние, что согласуется с нашей точкой зрения о том, что относительно тонкие факторы, влияющие на региональный инновационный потенциал, влияют на уровень регионального инновационного производства.

Чтобы проверить эффекты умеренности, мы сначала определяем средние показатели для инновационных конструкций, чтобы уменьшить потенциальную мультиколлинеарность, а затем вычисляем перекрестные продукты их показателей. Как показано в таблице 2, серия тестов показывает, что РИС Китая плохо работают в отношении взаимодополняемости между инновационными ресурсными факторами и контекстуальными факторами, основанными на системном мышлении.

Модель пути 4.9 показывает, что INNO_LINK оказывает статистически значимое, но отрицательное влияние на модерацию влияния EX_K_ ACQ на INNO_OUT на уровне 1\%. Когда CLUS_EN участвует вместе с INNO_LINK в процессе производства инноваций, модерация становится слабее и частично значимой на уровне $5 \%$. Модели путей 4.9 и 4.10 показывают, что INNO_LINK предотвращает вклад CU_K_STO в INNO_OUT, хотя признак эффектов модерации в двух моделях путей статистически отрицателен только на уровне 10\%. Кроме того, ключевым выводом является то, что CLUS_EN демонстрирует стабильно значительный и отрицательный эффект модерации на влияние INNO_IN на INNO_OUT в моделях 4.8, 4.10 и 4.12 на уровне $1 \%$. В нашей предпочтительной модели 4.12 CLUS_EN демонстрирует значительный и положительный эффект модерации на влияние CU_K_STO на INNO_OUT на уровне 5\%. Однако этот эффект умеренности не наблюдается в моделях путей 4.8 и 4.10. Фактически, в литературе велись дебаты об относительной эффективности использования перекрестных продуктов показателей и перекрестных продуктов агрегированных показателей. Те же результаты получены и в нашей выборке. Этот вывод обеспечивает частичную поддержку эффекта модерации CLUS_EN на влияние CU_K_STO на INNO_OUT.

Наши результаты показывают, что инновационная среда не является хорошим стимулом и стимулом для региональной инновационности Китая, хотя она доказала свою эффективность. Это не отделимо от разрыва между исключительными инвестициями в научно-техническую деятельность и отставанием в выполнении рамочных условий для региональных инноваций. Таким образом, эти впечатляющие инвестиции в ресурсы внесли значительный вклад в быстрый социально-экономический прогресс, достигнутый в Китае за последнее десятилетие, но он еще не привел к пропорциональному увеличению показателей инновационной деятельности (ОЭСР, 2008).

В исследовании предложена интегрированная аналитическая структура инновационных систем с помощью структурного подхода, который не только учитывает нелинейные и взаимодействующие характеристики инновационных систем, но и облегчает эмпирическое применение системного подхода к инновациям. Эмпирически, именно с помощью аналитических рамок мы определили эффективность различных функциональных конструкций, определяющих производительность РИС Китая (т.е. насколько хорошо эти функции выполняются во время функционирования РИС Китая) в период первой пятилетки, вступающей в 21 век. Для этой цели мы построили серию функциональных конструкций, основанных на расширенной рамочной системе производственной функции идеи Ромера (1990), предложенной Фурманом, Портером и Штерном (2002). Затем мы применили структурный подход для включения различных 
функциональных конструкций в интегрированную аналитическую структуру. Это позволяет нам всесторонне определить те ключевые функциональные конструкции, которые формируют или влияют на региональный инновационный процесс в Китае.

С точки зрения оригинальности, наша аналитическая структура позволяет эмпирически выявлять и диагностировать рабочее состояние инновационных систем с функциональной точки зрения в отличие от концептуальных или теоретических исследований в существующем исследовании. Это означает, что наша аналитическая база позволяет эмпирически исследовать инновационные системы, оценивая их «функциональность». Соответственно, наши результаты эмпирических исследований с использованием структурного подхода дают систематические доказательства специфики РИС Китая в отличие от конкретных показателей в существующих ограниченных эмпирических исследованиях.

Кроме того, наш подход к исследованию на основе PLS обеспечивает более надежные результаты для эмпирического исследования детерминант инновационных систем по сравнению с теми, которые основаны на OLS исследовательские инструменты, которые повреждены небольшими наборами данных, а также мультиколлинеарностью в существующих моделях.

В эмпирическом анализе серия регрессий PLS в первую очередь показывает, что университеты, правительства и программы, отраслевые спецификации и государственные исследовательские учреждения играют решающую роль в повышении регионального инновационного потенциала. Это, в частности, отражает хорошо выполняемые функции государственной политики Китая и специфичную для кластера среду в процессе региональной модернизации. Кроме того, установлено, что университеты, государственные научно-технические программы и государственные научно-исследовательские институты в основном вносят вклад в выпуск научных инноваций, в то время как специфическая для кластера среда отвечает за выпуск технологических инноваций.

Моделирование траектории предоставило убедительные доказательства того, что три основные конструкции, основанные на инновационных ресурсах, накопленный запас знаний, дополнительные инновационные ресурсы, а также внешние инновационные приобретения, демонстрируют значительный вклад в региональный инновационный выпуск Китая. Более того, мы также считаем, что большинство экзогенных движущих и поддерживающих структур также играют важную роль в реализации регионального инновационного потенциала Китая, и особенно специфическая для кластера среда, и общая инновационная среда (финансовая, коммуникационная и маркировочная среда) будут способствовать и стимулировать региональный инновационный выпуск Китая. Однако две конструкции, инновационная сложность и инновационная связь, имеют надежные, но значительно отрицательные коэффициенты, которые оказывают неожиданное влияние на инновационный результат. Неожиданный эффект инновационной изощренности, связанный с инновационным потенциалом фирмы, показывает, что РИС Китая не являются несовершенными, и, по крайней мере, региональная инновационная система, ориентированная на фирму, на самом деле не сформировалась в большинстве провинциальных регионов Китая. Рассмотренные связи университетов с венчурным капиталом, демонстрирующие худшие результаты в области инноваций, подтверждают нынешнюю плохую ситуацию с системой высшего образования и системой венчурного капитала в Китае. Цель университетов Китая явно состоит в том, чтобы создать ряд университетов мирового класса, которые были бы менее вовлечены в то, что сейчас должно быть в первую очередь задачей делового сектора (ОЭСР, 2008). Кроме того, существует острая нехватка капитала для финансирования новых предприятий, а также нехватка как экспертных знаний, так и необходимых правовых и нормативных условий для адекватно функционирующей системы венчурного капитала (ОЭСР, 2008). Плохая работа китайской системы венчурного капитала является неизбежным результатом.

Взаимосвязи взаимодействия между инновационными ресурсами и факторами инновационной среды неэффективно проявляются с помощью ряда тестов на смягчающие эффекты. Как упоминалось ранее, одна из ключевых причин заключается в том, что скорость разработки рамочных условий отстает от темпов инвестиций в научно-технические разработки на региональном уровне. Хотя Китай пытается способствовать переходу от инновационной 
системы, ориентированной на более ПРО (государственные исследовательские организации), к инновационной системе, ориентированной на фирмы, нынешние инструменты политики и управления для поощрения инноваций в более плановой экономике демонстрируют большую неэффективность (ОЭСР, 2008), поскольку стимулирующие эффекты рамочного условия хорошо работают в контексте рыночной системы. Это напоминание о том, что правительства на всех уровнях должны изменить свои акценты с нынешнего резкого увеличения инвестиций в научно-технические разработки на равномерное улучшение рамочных условий для инноваций. Только таким образом инвестиции в научно-технические разработки могут быть переведены в пропорциональное повышение эффективности инноваций, т.е. могут быть возвращены более эффективным способом с точки зрения инновационной продукции. Мы считаем, что их совершенствование могло бы создать необходимые условия для функционирования модели открытых инноваций, в которой местные инновационные возможности и интенсивные иностранные инвестиции в НИОКР могли бы взаимно усиливать друг друга. Это также оказывает решительную поддержку заявлению ОЭСР (2008) о том, что улучшение широкого спектра рамочных условий для поддержки рыночных инноваций должно стать приоритетом в реализации долгосрочного и среднесрочного стратегического плана в области инноваций.

Наши выводы имеют важные политические последствия. Во-первых, роль университетов в инновационной системе, ориентированной на фирмы, должна быть изменена, чтобы она служила практическому применению. В каждом регионе следует поощрять и продвигать инновационную модель сотрудничества между промышленностью и университетами, которая лежит в основе наиболее инновационных сетей и кластеров (ОЭСР, 2008). Во-вторых, функционирующая система венчурного капитала должна быть построена путем совершенствования ее модели управления. Согласно исследо- ванию ОЭСР (2008), отечественные венчурные компании были созданы правительством на национальном или провинциальном уровне и управляются государственными должностными лицами, которые не всегда обладают [6] достаточными техническими, коммерческими или управленческими навыками. В-третьих, правительствам на всех уровнях следует изменить свои акценты с нынешнего резкого увеличения инвестиций в научно-технические разработки на равномерное улучшение базовых условий для инновационной производительности. Вчетвертых, промышленным предприятиям Китая необходимо развивать связи между наукой и промышленностью и использовать ресурсы НИОКР сектора высшего образования и научноисследовательских институтов для укрепления их потенциала в области НИОКР за счет сотрудничества и распространения технологий.

Наше исследование имеет определенные ограничения и нуждается в расширении. Кроме того, инновации, не связанные с НИОКР, в основном включающие организационные и управленческие инновации (ОЭСР и ЕВРОСТАТ), следует рассматривать в связи с их вкладом в региональный инновационный потенциал. В то же время, может быть интересной идеей реализовать эту тему в рамках эффективности или производительности, учитывающей такие неизмеримые детерминанты, как статистический шум и факторы окружающей среды. Кроме того, необходимо внедрить международный сравнительный анализ между подразделениями региона и страны, поскольку несколько китайских провинций или даже муниципалитетов в настоящее время демонстрируют более высокие показатели НИОKР, чем несколько стран ОЭСР (OECD).

Таким образом, предложенная модель во многом отражает межотраслевые и межрегиональные диспропорции инновационного развития в КНР. При этом предложенная является адаптивной к учету дополнительных показателей и может быть использована при анализе НИС других государств для выявления роли региональных инновационных систем. 


\section{Библиографический список}

1. Пястолов С.М. 2013. 02. 020-021. Структурный подход в исследованиях региональных инновационных систем на примере Китая (сводный реферат) // Социальные и гуманитарные науки. Отечественная и зарубежная литература. Сер. 8, Науковедение: Реферативный журнал. - 2013. - № . 2.

2. Furman J.L., PorterM.E., Stern S. The determinants of national innovative capacity // Research policy. - 2002. - T. 31. - № . 6. - C. 899-933.

3. Cheung K., Ping L. Spillover effects of FDI on innovation in China: Evidence from the provincial data // China economic review. - 2004. - T. 15. - № . 1. - C. 25-44.

4. Romer P. M. Endogenous technological change // Journal of political Economy. - 1990. - T. 98. - № . 5, Part 2. - C. S71-S102.

5. Шелупин С.В. Китай: от длительного к устойчивому развитию. Инновационный аспект // Актуальные проблемы гуманитарных и естественных наук. - 2009. - № . 11.

6. Хасунщев И. М. Использование опыта стран брик при формировании национальной инновационной системы России // Вестник университета. - 2011. - № . 26. - С. 235-238. 